\title{
Review and Prospect of the Preventive Effect of "Supplementing Qi and Nourishing Yin, Activating Blood Circulation and Detoxifying" on Diabetes Mellitus Complicated with Acute Coronary Syndrome
}

\author{
Fu Xianzhao, ${ }^{1}$, Huang Zhenfeng ${ }^{2}$, Huang Wenhua ${ }^{1}$, Tan Wanli ${ }^{2}$, Li Chunyan ${ }^{1}$, Pan Xingshou ${ }^{1}$, \\ Huang Qingli ${ }^{1}$, Ban Fudu ${ }^{1}$ \\ ${ }^{1}$ Clinic Medical College of Youjiang Medical National College, Baise, China \\ ${ }^{2}$ Napo County People's Hospital, Napo, Baise, China \\ Email address: \\ 1620100638@qq.com (Fu Xianzhao) \\ ${ }^{*}$ Corresponding author
}

\section{To cite this article:}

Fu Xianzhao, Huang Zhenfeng, Huang Wenhua, Tan Wanli, Li Chunyan, Pan Xingshou, Huang Qingli, Ban Fudu. Review and Prospect of the Preventive Effect of "Supplementing Qi and Nourishing Yin, Activating Blood Circulation and Detoxifying” on Diabetes Mellitus Complicated with Acute Coronary Syndrome. American Journal of Clinical and Experimental Medicine. Vol. 6, No. 4, 2018 , pp. 87-93. doi: 10.11648/j.ajcem.20180604.11

Received: June 2, 2018; Accepted: June 19, 2018; Published: July 12, 2018

\begin{abstract}
At present, the prevalence of diabetes is on the rise, while the cardiovascular disease is a major complication of diabetes mellitus. Acute coronary syndrome (ACS) is a severe form of coronary heart disease. Compared with nondiabetic patients, there are more difficulties and troubles in deal with diabetic ACS patients with more serious condition. Although percutaneous coronary intervention (PCI) played a certain role on recanalization for coronary artery, the problems facing PCI are still serious for the diabetic ACS patients after the operation. Traditional Chinese medicine (TCM) confirms that the etiology and pathogenesis of diabetic ACS are deficiency in Qi and yin, and accumulation in stasis and toxin. Through clinical observation and retrospective analysis, We found that the "Supplementing Qi and nourishing Yin, activating blood circulation and detoxifying", which simplified the treatment prescription, are integrated treatment of multiple links, multiple path ways, and multiple targets for the diabetic ACS, and are the unique advantages of the holistic concept, syndrome differentiation for treating and multi-levels intervention of TCM full practice in the comprehensive prevention and treatment for diabetic ACS.
\end{abstract}

Keywords: Diabetes Mellitus, Coronary Heart Disease, Acute Coronary Syndrome, Supplementing Qi and Nourishing Yin, Activating Blood Circulation and Detoxifying

\section{Introduction}

At present, the prevalence rate of diabetes is on the rise, which in China is up to $9.79 \%$, higher than $6.4 \%$ of the world average [1], and China is the most populous country in the world, which makes China bear huge diabetes burden [2]. Cardiovascular disease is the major complication of diabetes. As early as 2001, America Cholesterol Education Program for Adult Treatment Panel III (CEP-ATP III) made it clear that "Diabetes and coronary heart disease (CHD) have the same risk", that is, diabetics with no CHD have the same risk as non diabetic patients who have had CHD, and have the same chances to suffer from new cardiovascular events within 10 years [3]. Acute coronary syndrome (ACS) is a series of clinical manifestations caused by inflammatory reaction in the coronary arteries, resulting in atherosclerotic plaques unstable or even ruptured [4]. ACS is a critical form of CHD, which is divided into unstable angina, non-ST segment elevation myocardial infarction, and ST segment elevation myocardial infarction, based on whether the electrocardiogram has ST segment persistent elevation and whether the blood has myocardial necrosis or not. The acute cardiovascular events triggered by ACS, whose incidence and mortality have surpassed that of cancer, has become the number one killer of 
global human life [5]. Compared with non-diabetic patients, diabetes mellitus complicated with ACS is more serious, with more contradictions and difficulties to treat. With the improvement of people's living standard, the prolongation of human life span and the aging of the population, China will again meet the rising of diabetes and cardiovascular diseases. Therefore, it is urgent to carry out the prevention and treatment of diabetic ACS, reduce the incidence of terminal events of cardiovascular disease, and improve the patients life quality. Exploring the prevention and treatment strategy for diabetic ACS has double significance both in treating diabetes mellitus and in preventing the arrival of the peak of cardiovascular disease.

\section{Compared with Non-diabetic Patients, Diabetic ACS has Greater Harm and Its Treating Methods Lag Behind}

The main cause of death and disability in diabetic patients is the complication of cardiovascular disease [6], whose prevalence and mortality rate are 2-4 times higher than those of non-diabetic patients. Diabetes is an independent risk factor for atherosclerosis (AS), which can occur either before or after AS, causing and aggravating AS. Currently, AS is considered to be the leading cause of death in diabetics [7]. The pathogenesis of diabetes and CHD has the same soil: chronic inflammation and insulin resistance. On the one hand, the increase of sugar oxidation product, glycosylated products and lipid oxidation products caused by lipid metabolism disorder can lead to vascular endothelial dysfunction, accelerate the formation of foam cells and speed up the development of AS; on the other hand, hyperglycemia can enhance oxidative stress, which may have multiplicative effect relationship with other cardiovascular risk factors such as hyperlipidemia, hypertension, and smoking, leading to endothelial damage and quicken the progress of AS [8-9]. And because multiple vascular stenosis and microvascular lesions coexist in diabetic CHD, once ACS occurs, it may lead to extensive ischemia of the myocardium and insufficiency of the heart blood supply. Therefore, compared with non-diabetic patients, the conditions of diabetic CHD are more serious, complex and progress rapidly, with more contradictions and difficulties to treat [10]. After 1980s, the incidence and fatality rate of CHD in the general population are decreasing. However, among the diabetes, with the increase of their prevalence and relative delayed prevention and treatment, the prevalence and mortality of diabetic cardiovascular diseases are increasing, and about $80 \%$ diabetic patients die from cardiovascular complications, with CHD accounting for $75 \%$ [11]. All this indicate that current diabetic CHD have not been effectively controlled, especially that the approach for ACS prevention and controlling are relatively hysteretic, fully showing the necessity of strengthening diabetic ACS prevention and control research.

\section{Problems in Treating Non-revascularization Diabetic ACS Patients}

Research found that nearly 50\% ACS patients failed to revascularization therapy, so this group of patients (diabetic ACS patients in particular) have higher mortality rate. Type 2 diabetes are often aggregated with $\mathrm{AS}$, and most of the patients are insidious onset, with no obvious symptoms and easily missed diagnosis, especially those aged patients with long course of disease. Due to the lack of typical angina, 30\% patients with diabetic coronary heart disease were not found early, and the ACS was the first pattern of manifestation. Only during the physical examination, abnormal Q waves were found in the electrocardiogram before myocardial infarction was detected [10]. In some cases, when ACS occurs, its symptoms are easily drowned by symptoms of other accompanying diseases and lead to be misdiagnosed [12]. In diabetic patients with $\mathrm{CHD}$, the coronary artery is usually multivessel and diffuse lesion, which can cause the ECG vector to cancel each other [13]. There is low positive rate for the diagnosis of multivessel disease with electrocardiogram. Moreover, the activity of sodium potassium pump and calcium pump in diabetic cardiomyocytes is abnormal, and the transmembrane voltage change is small during repolarization, which easily cause electrocardiogram changes blurred [14]. Therefore, there is no good relationship between clinical manifestations, electrocardiogram and coronary angiography. Sometimes, diabetic ACS could display complex conditions such as "normal electrocardiogram" and painless myocardial ischemia, which are often easily ignored and delayed for diagnosis and treatment, and miss the best time window for revascularization. Moreover, revascularization is difficult for multiple diffuse coronary artery disease, so there is little chance for diabetic CHD vessel reconstruction. In addition, the county hospitals in most remote areas do not have the conditions for revascularization, and the simple standardized western medicine still has a large residual risk [15]. Therefore, the diabetic ACS patients with non-revascularization are faced with seeking the combination of traditional Chinese and Western medicine for intensified prevention and treatment.

\section{The Problems Faced with Diabetic ACS After Revascularization}

Since 1977, Cruentzig, a Swiss scientist, successfully used percutaneous coronary intervention (PCI)for the treatment of coronary stenosis, created a new age for the treatment of coronary heart disease in medical history, PCI has become an effective method for the treatment of CHD at full speed, and been widely used in reperfusion or revascularization for CHD. PCI can open infarct blood vessels and restore myocardial perfusion, which can obviously improve the symptoms and prognosis of ACS patients, reduce the mortality, and change the pattern of relying on drug therapy and coronary artery bypass grafting by surgical thoracotomy. However, the prognosis of 
diabetic CHD patients after PCI is different from that of non-diabetic patients. In general, non-diabetic patients have a compensatory movement of the non-infarcted vascular after the onset of myocardial infarction, while in diabetic patients, the vascular compensatory movement is weakened due to the multiple vascular and myocardial microvascular lesions [16]. Therefore, even if PCI is suitable and timely, infarcted blood vessels are opened to restore myocardial perfusion, and the patient has a temporary remission, but the progress of cardiac remodeling is faster than that of non diabetic patients (4 years death rate was $25.9 \%$ vs $14.5 \%$ ) [17]. Moreover, after successful emergency intervention of the infarct related vessels in patients with multiple coronary artery disease, even if the preventive intervention of a non-infarct narrow vessel can significantly reduce the risk of CHD rehospitalization treatments, it does not reduce the risk of recurrent myocardial infarction and cardiac death, on the contrary, it increases the number of stent implantation and total medical cost [18]. Therefore, a series of problems facing the revascularization of diabetic ACS have also become an insurmountable obstacle to the development of interventional therapy.

\section{Diabetic ACS “Qi-yin Deficiency, Blood Stasis and Toxin Accumulation" Pathogenesis Analysis}

Ancient doctors in China have a great deal of discussion about "All accumulated pathogeny would turn into toxiin with the passing of time", «Su Wen`wu chang zheng da lun: Plain Questions-Great Theory on Shifting and Interaction of Five Phases» "Toxic pathogenesis initiate from all five elements of violent Qi"; In the «synopsis of the golden chamber», Eastern Han Dynasty medical expert Zhang Zhongjing classified the toxins into Yang toxin (heat toxin, clear toxin) and Yin toxin (cold toxin, damp toxin, dryness toxin);Song dynasty Chen wuze «San yin Ji-Bing zheng Fang Lun: Treatise on Three Categories of Pathogenic Factors» put forward the theory of "Three causes cause diseases", dividing complex pathogenic factors into three categories, which are external causes, internal causes and neither internal nor external causes, holding "The main causes of the diseases are no more than three causes", assuring that the three causes can cause disease alone, also can be combined to cause disease, and that no matter what pathogeny causes acts on the human body, will initiate the struggle between healthy Qi and pathogenic Qi, leading to pathogen accumulation, and for long time turning into toxin. Qing dynasty You Zaijing «Jin Kui Yao Lue Xin Dian:the Essence of Synopsis of the Golden Chamber» "The toxin is the pathogen stagnated and undissolved". Diabetes mellitus (Xiao Ke) and coronary heart disease (chest obstruction) coexist in the development and evolution on the basis of Yin deficiency and dry heat accumulation, whose pathogenesis involves the following aspects: (1) Yin deficiency and dryness-heat accumulation can turn the liquid into phlegm, then phlegm and stasis block the heart choroid and make chest pain; (2) Yin deficiency and dryness-heat cause Qi-Yin deficiency, and Qi-deficiency can not drive blood circulation, then blood stagnate and become stasis, block the heart vascular, and make chest pain; (3) The long onset of Qi-Yin deficiency can lead to Yin-Yang deficiency, then cold accumulation and heart Yang incapability cause blood stasis, obstruct the heart vascular, and result in chest pain. As a result, Xiao $\mathrm{Ke}$ and chest pain can lead to accumulation of phlegm dampness, blood stasis, and dry heat, and eventually turn to toxin. The pathogenesis of diabetes mellitus complicated with coronary heart disease can be summed up as "Qi-Yin deficiency, blood stasis and toxin accumulation". The heat-toxin can lead to changeable disease with its intensive character, and often evolve into all kinds of danger, such as ACS, which can kill people. Just as the "Han liang pai: Cold and cool academic school Liu Wan Su, one of the four great physicians in Jin-Yuan dynasty, said "The cause of sudden morbidity and death is the onset of the fire". Therefore, heat-toxin is ascribed the main pathogenesis of ACS, which reflects the essence of ACS more completely, compared with the "Yang wei Yin xian: depression of Yang Qi and accumulation of Yin cold" summarized by Zhang Zhongjing «Jingui Yaolue: Synopsis of Golden Chamber».

\section{Analysis of Diabetic ACS Etiology and Pathogenesis After PCI}

The physique theory of traditional Chinese medicine (TCM) divides human constitution into nine kinds of clinical physique, which are normal quality, Qi deficiency, Yang deficiency, Yin deficiency, phlegm dampness, damp heat, Qi stagnation, blood stasis and special quality, believing that the nature and pathogenetic process of diseases are closely related to the physique of the patients, and often susceptive to certain pathogenic factors or diseases. The physique specificity determines the state of reaction, the nature of disease and the trend of its development. It is one of the TCM characteristics to attach importance to human physique and its differences. «Neijing Ling shu·Shou yao Gan grou» "People's constitution physique is inherent, with all kinds of rigid, soft, weak, strong, short and long, and Yin Yang" clearly pointed out that people can display individual differences in the process of life. In «shang han za bing lun: treatise on febrile and miscellaneous diseases», Zhang Zhongjing made further elaboration on the relationship between constitution and pathogenesis, syndrome differentiation, treatment and prognosis. People's native endowment and acquired factors determine their physical fitness. Modern medical research also shows that the incidence of diabetes and coronary heart disease are mostly related to genetic constitution. Therefore, although the stent placement for diabetic ACS patients has a certain effect on the reopening of the coronary arteries, it does not remove the lesions, and the atherosclerotic plaque still exists. What is more, stent implantation can cause a new damage to the coronary artery as well, which will aggravate the inflammation and edema of the local lesion and aggravate the blood stasis. The essences of the deficiency of Qi and Yin and 
the stagnation of phlegm and stasis toxin, which block the coronary artery, are still the etiology and pathogenesis of ACS after PCI.

\section{The Theoretical Basis of "Nourishing Yin, Supplementing Qi, Activating Blood Circulation and Detoxicating" Therapy to Prevent Diabetic ACS}

After the prolonged onset of Xiao Ke complicated chest pain, Yin deficiency and dry-heat can cauterizate fluid and turn it into phlegm, and Qi deficiency can contribute to blood coagulation and stasis, subsequently, the phlegm and blood stasis, when accumulated for a long time, will turu into toxin. Therefore, Qi Yin deficiency and blood stasis are the main pathogenesis of Xiao Ke chest pain, which should be treated with nourishing Yin and nourishing Qi, activating blood and detoxifying [19]. In fact, in Qing Dynasty, the heat-clearing and detoxicating prescription has been applied in clinical heartache treatment, such as, medical expert Chen shiduo «Medica encyclopedia·Yu Han Differentiation·Heartache» found "xie huo shen dan: Clearing fire Panacea", Applying "clearing heat and detoxifying therapy", using large doses of Gardenia and Aspidium succeed in treating heartache. Scientific research has shown that AS is a process of artery post-injury inflammation, leaading inflammatory cytokines accumulation and metabolic activity increment in the plaque, causing inflammatory response to release heat. In stable angina, unstable angina and acute myocardial infarction after PCI, the temperature of local plaque was higher than that of the peripheral coronary artery, and it was positively correlated with the plaque instability [20]. The inflammatory reaction is similar to the characteristics of heat toxin, for the inflammatory infiltrating and temperature rising is a manifestation of Yang heat, which suggests that the drugs with clearing heat-toxin effect may play a potential protective effect on the heart by anti-inflammatory. Therefore, the treatment for diabetic ACS patients, and their perioperative or postoperative long-term treatment should focus on the basic pathogenesis of heat toxin, and attach importance to clearing away heat and toxic substances. If we adhere to the etiology and pathogenesis of "Yang wei Yin xian: depression of Yang Qi and accumulation of Yin cold" chest or heart pain, and focus on "warming up" treatment, select radix aconiti, aconitum warm toxic drugs and so on, the treatment of diabetic ACS can only be antagonistic. Therefore, the "Supplementing Qi and nourishing Yin, activating blood circulation and detoxifying" therapy is suitable for diabetic ACS, which can be used for diabetic ACS patients who lost the opportunity or not suitable for PCI, and which can also be used for continuing treatment in patients after PCI. "Supplementing Qi and nourishing Yin" is the permanent treatment for the root cause of the disease, "activating blood circulation and detoxifying" is the temporary cure for acute symptoms at present. Temporary and permanent combination can make Yin fluid, Qi and blood sufficient, make stasis and toxin disbanded, finally make blood vessels unobstructed, and provide a new way to treat diabetic ACS.

\section{The Practical Exploration of "Nourishing Yin, Supplementing Qi, Activating Blood Circulation and Detoxicating" Therapy to Diabetic ACS}

Based on "co-existence of deficiency of both Qi and Yin, blood stasis and toxin accumulation" as the basic pathogenesis of diabetic ACS, accordingly, "Nourishing Yin, supplementing Qi, Activating blood circulation and Detoxicating" is its main therapeutic rules. Huoxue Jiedu Jiangtang formulation (HJJF)consist of turtle shell, ginseng, astragalus, ophiopogon japonicus, fructus corni, radix rehmanniae, rhubarb, rhizoma coptidis, salvia miltiorrhiza, peach kernel, cortex moutan, yam and fructus schisandrae chinensis, in which, ginseng, ophiopogon root, schisandra are $\mathrm{Li}$ Gao(one of the four major medical scientists in Jin Yuan Dynasty)《Endogenous and Exogenous differentiation theory» Shengmai Powder ingredients, possessing nourishing Yin, tonifying Qi, generating fluid, and nourishing heart; cornus, yam and rehmannia are Ming Dynasty doctor Zhang Jingyue «Jing Yue Quan Shu: Jingyue' s Complete Works» Zuo Gui pill ingredients with effect of nourishing kidney, filling Yin and suppressing Yang, for treating true Yin deficiency; turtle shell, rhubarb, peach kernel and cortex moutan are Eastern Han Dynasty medical expert Zhang Zhongjing's "Turtle shell pills" ingredients for treaing “Accumulation”. Qing Dynasty physician Ye Tianshi, attached importance to using animal drugs, explicating "Take advantage of the flexible characteristics of rapid flying in insects or ants,......, Walking can come down or scattered, thus blood can not coagulate, Qi can dispersed", and pointing out that the curing of obstinate diseases caused by phlegm-blood stasis can only be treated by creature drugs with activating and dissipating stasis effects. HJJF prescription selects turtle shell to nourish kidney Yin, soften and dissipate stagnation, and clear away asthenia heat, catering to the diabetic ACS constitution with yin-deficiency, dry-heat, stasis-toxin; Astragalus membranaceus combined with ginseng, can supplement Qi and strengthen the vital energy, invigorate $\mathrm{Qi}$ and generate blood, making toxin floated and dissipated; rhizoma coptidis coordinated with rhubarb can eliminate heat-toxin and dispelling dampness; salvia miltiorrhiza combined with peach kernel and cortex moutan, can promote blood circulation and dissipate blood stasis. All prescriptions, supplementing and attacking in combination, share the effects of "Supplementing Qi, nourishing Yin, activating circulation, eliminating toxin, softening plaques and dissipating stasis".

Adopting the prospective method, previous studies selected the diabetic ACS patients who had undergone PCI for experiment. Patients were randomly divided into a observation group and a control group by a computer. On the 
basis of western medicine therapy, the observation group additionally received HJJF, while the control group was given standardized western medicine only. The effect of HJJF on cardiac remodeling and cardiac function prognosis of patients after PCI was investigated. The indexes include, morphological indexes of left ventricle: left ventricular end-systolic diameter (LVESD), left ventricular end diastolic diameter (LVEDD), left ventricular posterior wall thickness (LVPWT), left ventricular end-diastolic ventricular septal thickness (LVEST), left ventricular function index: left ventricle ejection Fraction (LVEF), the maximum blood flow velocity of early diastolic/atrium late diastolic (E/A), stroke volume (SV), cardiac output (CO), cardiac index (CI), Left ventricular fractional shortening $(\Delta \mathrm{FS})$, serum Ang II, pro-inflammatory factors, cardiac function and clinical efficacy and so on. The results showed that the total effective rate of the observation group was higher than that of the control group. The decreasing levels of Serum inflammatory mediators [interleukin-6(IL-6), C-reactive protein (CRP), tumor necrosis factor-a $(\mathrm{TNF}-\alpha)]$ and Ang II are superior to that of the control group. Left ventricular morphological indexes LVEDD and LVESD in observation group were lower than those in control group, while left ventricular function indexes LVEF, E/A, SV, CO, CI and 1eft ventricular fractional shortening $(\triangle \mathrm{FS})$ were higher than those in control group. This research shows that, by antagonizing inflammatory response and regulating the leve $\Delta 1$ of Ang II, HJJF can inhibit cardiac remodeling, enhance heart function and improve the prognosis of diabetic ACS patients after PCI operation, and that the curative effect is better than that of routine treatment of Western Medicine [21].

The same research methods were used to randomly allocate diabetic ACS patients who had no revascularization for various reasons into observation group and control group for experiment, comparing two groups TCM clinical syndromes, the frequency and duration of chest suppression and chest pain, observing the palpitation, shortness of breath, fatigue and the total syndrome score, surveying their electrocardiogram and the levels of pro-inflammatory and anti-inflammatory factors. The results showed that the duration of chest suppression and pain, fatigue and the total syndrome score in observation group (HJJF + Western Medicine)were significantly lower than those in the control group (Western Medicine); the levels of pro-inflammatory cytokines CRP, IL-6 and TNF- a were significantly lower than that in the control group, while the levels of anti-inflammatory factors IL-10 and adiponectin (ADPN) were higher than that in the control group; the number of ECG ST segment decline, T wave low-lying, and T wave inversion were significantly lower than that in the control groups. It is proved that HJJF can effectively regulate the anti-inflammatory/ pro-inflammatory balance and improve the diabetic ACS myocardial blood supply [22].

Although "Nourishing Yin, supplementing Qi, Activating blood circulation and Detoxicating" is the therapy for diabetic heart disease, but the stasis toxin has the degree of severity. ACS has serious stas $\Delta$ is toxin, so it is treated with HJJF, while in diabetic patients complicated with chronic heart failure (CHF), the stasis toxin reaction is relatively mild and the deficiency of Qi and Yin is prominent, so Jiangtang Shuxin decoction (JTSXD) (reducing turtle shell and purgative rhubarb from HJJF) was chosen to treat diabetic CHF, and the therapy do not depart from "Nourishing Yin, supplementing Qi, Activating blood circulation and Detoxicating". The results showed that, compared with conventional western medicine treatment, JTSXD combined with conventional treatment could more effectively alleviate ventricular filling pressure, reduce the left ventricular end diastolic volume (LVEDVL) morphological indexes, cut down the plasma level of Ang II and brain natriuretic peptide (BNP), increase SV, CO, LVEF, E/A, improve cardiac function (increase the 6-minute walking distance), and so on. It can be seen that "Nourishing Yin, supplementing Qi, Activating blood circulation and Detoxicating" therapy can effectively reduce insulin resistance in diabetic patients with CHF, control blood glucose, correct lipid metabolism disorders, regulate cardiac energy metabolism, improve cardiac remodeling, inhibit the renin angiotensin aldosterone system (RASS) activated by heart failure and reduce the secretion of BNP in the ventricle, thus improve the heart function [23].

In animal experiments, skeletal muscle specific insulin-like growth factor-1 receptor deficient mice (this rat has the characteristics of type 2 diabetes at fifth weeks of birth) were selected to study. The results showed that, compared with the model group, the JTSXD, which have the "Nourishing Yin, supplementing Qi, Activating blood circulation and Detoxicating" effect, could obviously inhibit the cardiac myocytes ERS, reduce (endoplasmic reticulum stress, ERS) marker molecules (glucose-regulated protein 78, GRP78) and (CCAAT/Enhance-Binding Protein Homologous Protein, CHOP) mRNA transcription and its functional proteins expression, inhibit the CHOP ERS apoptosis pathway, reduce cardiac myocytes apoptosis, alleviate the myocardial fibrosis, and thus reverse the cardiac remodeling [24].

\section{Conclusion and Prospects}

Although the guidelines for clinical diagnosis and treatment of coronary heart disease have been established both at home and abroad, there are still individual differences in guiding clinical practice, especially in patients with diabetes. Compared with non-diabetic patients, diabetes can make diabetic coronary heart disease worsened, resulting in poor prognosis and low survival rate. With the continuous development of clinical research, the maturation of the treatment technology and the improvement of the stent implanted materials, the indications of PCI are constantly expanding, but PCI cannot change patients' genetic constitution, insulin resistance condition, and the disorder of glucose and lipid metabolism. For diabetic ACS, the problems facing post stent implantation are still serious. Through clinical observation and retrospective analysis, the "Nourishing Yin, supplementing Qi, Activating blood circulation and Detoxicating" therapy in the people with 
diabetic coronary heart disease catch hold of the stasis-heat toxin pathogenesis in diabetic ACS, simplify the treatment plan, exert its whole regulation treatment of multi-link, multi-way and multi-target, embody TCM unique advantage of the overall concept, the syndrome differentiation and treatment, and the multi link intervention, which playing an important role in the comprehensive treatment of diabetes ACS, and supplementing to the routine medical treatment in modern medicine. However, further clinical trials are needed to determine the exact clinical efficacy, so we still need a multi center, large sample, randomized controlled clinical trial, a long term follow-up study, and a thorough study of the pharmacological mechanism of Chinese medicine, elaborate TCM "syndrome differentiation and treatment, dynamic adjustment, overall intervention" for diabetic ACS prevention and treatment, and obtain good efficiency.

\section{Acknowledgements}

The research is supported by National Natural Science Foundation of China (81460698), Guangxi Emphasis Research and Development Program (2017AB45042), Guangxi Natural Science Foundation (2015GXNSFAA139221), Guangxi Inheritance and Innovation Subject of TCM and National Medicine (GZLC16-51) and Guangxi College Students Innovative and Creative Project (201510599021).

\section{References}

[1] YIN Yan-hua, SUN Hai-yan, ZHAO Li, et al. Clinical analysis of glucose and lipid metabolism in elderly hospitalized patients with type 2 diabetes [J]. Chin J Diabetes, 2015, 23(5): 390-393.

[2] MU Yi-ming, JI Li-nong, YANG Wen-ying, et al. Chinese expert consensus on management of postprandial hyperglycemia for type 2 diabetes mellitus [J]. Chin J Diabetes, 2016, 24(5):385-389.

[3] Expert Panel on Detection, Evaluation, and Treatment of High Blood Cholesterol in Adults. Executive Summary of the Third Report of the National Cholesterol Education Program (NCEP) Expert Panel on Detection, Evaluation, and Treatment of High Blood Cholesterol In Adults (Adult Treatment Panel III) [J]. JAMA, 2001, 285(19):2486-2497.

[4] YANG Li, GU0 Yu-xuan, XU Fei-li. The correlation of Hcy, hs-CRP and acute coronary syndrome risk stratification [J]. Chinese Journal of Clinical Pathologist, 2014, 6 (4):212-215.

[5] Mozaffarian D, Benjamin EJ, Go AS, et al. Heart disease and stroke statistics-2015 Update: a report from the American Heart Association [J]. Circulation, 2015, 131:e29-e322.

[6] GAO Qing-ge, ZHU Chao-yu, WEI Li, et al. Characteristics of vascular diseases in hospitalized patients with type 2 diabetes and the influencing factors [J]. Chin J Diabetes, 2015, 23 (3): 203-206.

[7] The endocrinology section of the Chinese Medical Association. The classificatory prevention guidelines of atherosclerotic cerebral and cardiovascular diseases in chinese adults With diabetes [J]. Chin J Endocrinol Metab, 2016, 32(7):540-545.
[8] Liu Ming-ming, Li Bing-wei, Wang Bing, et al. Microcirculation dysfunction and diabetes mellitus [J]. Chin J Endocrinol Metab, 2015, 31 (5): 471-473.

[9] PENG Xiao-ren, ZHAO Yan-fang, ZOU Da-jin, et al. The role of diabetes mellitus as a risk factor of acute myocardial infarction [J]. Chin Crit Care Med, 2011, 23 (6): 322-328.

[10] GAO Yang, LU Bin, HOU Zhi-hui, et al. Coronary atherosclerosis lesion features in coronary artery disease patients complicating with metabolic syndrome [J]. Chin J Cardio, 2013, 41(11): 935-939.

[11] Expert group on early screening and management of glucose metabolism disorders in cardiovascular medicine. Expert consensus on early screening and management of impaired glucose metabolism in cardiovascular medicine [J]. Chin $\mathrm{J}$ Intern Med, 2012, 51 (7): 574-577.

[12] LU Ying, WANG Tao, HUA Li-min. Investigation of Clinical Characteristics in Patients with Coronary Heart Disease and Diabetes Mellitus [J]. Chinese Journal of Trauma and Disability Medicine, 2008, 16(5):27-28.

[13] ZHANG Wen-bo. ECG diagnosis clues and misunderstandings [M]. Beijing: People's Military Medical Publishing House, 2010:34-44, 90-92.

[14] ZHOU Yun-ling. Causes and preventions of misdiagnosis of 78 patients with acute coronary syndrome [J]. Practical Journal of Clinical Medicine, 2008, 5(6): 78-80.

[15] YUAN Fei, SONG Xian-tao, LU Shu-zheng, et a1. Percutaneous coronary intervention in mainland China in 2008: register results $[J]$. Chinese Journal of Cardiology, 2010, 38 (7): 629-632.

[16] XIAO Jian-yong, ZHANG He-nan, CAO Lu, et a1. An analysis of relationship between the severity of coronary artery lesion and risk factors of cardiovascular events in Tianjin [J]. Chinese Critical Care Medicine, 2013, 25(11):650-654.

[17] Michaelael TJ, Aristidis V. Diabetes and cardiovascular disease [M]. Sun FL translation. Ji Nan: Shandong science and technology press, 2008, 430-431.

[18] Zhang Jing, Wang Qing-sheng, Yang Hong-mei, et al. Evaluation of different revascularization strategies for patients with acute myocardial infarction with lesions of multiple coronary arteries after primary percutaneous coronary intervention and its economic evaluation [J]. Chinese Critical Care Medicine, 2015, 27(3):169-174.

[19] FU Xian-zhao, XU Jing, HUANG Wen-hua, et a1. Strategy of Stasis-resolving and Detoxification on Diabetic Coronary Heart Disease [J]. JETCM, 2014, 23(11):2024-2027.

[20] Toutouzas K, Drakopoulou M, Stefanadi E, et al. Intracoronary thermography: does it help us in clinical decision making [J]. J Interv Cardiol, 2005, 18(6): 485-489.

[21] FU Xian-zhao, XU Jing, LI Chun-yan, et al. Effect of Huoxue Jiedu Jiangtang formulation on heart remodeling in diabetic patients with acute coronary syndrome after PCI [J]. Chin J TCM WM Crit Care, 2016, 23(2): 117-121.

[22] FU Xian-zhao, XU Jing, LI Chun-yan, et al. Effects of Huoxue Jiedu Jiangtang formulation on the balance between pro-and anti-inflammatory cytokines in non-revascularization patients with diabetic acute coronary syndrome $[\mathrm{J}]$. Chin $\mathrm{J}$ TCM WM Crit Care, 2016, 23(1): 6-10. 
[23] FU Xian-zhao, HUANG Yue-feng, WANG Qing-li, et al. Effect of Jiangtang Shuxin decoction on diabetic patients with chronic heart failure:a prospective randomized controlled study [J]. Chin J TCM WM Crit Care, 2017, 24 (2): 123-126.
[24] FU Xian-zhao, YU Rong, CHENG Xi-hua, et al. Effects of Jiang Tang Shu Xin Recipe on MKR diabetic mice endoplasmic reticulum stress-related apoptosis signal CHOP and myocardial remodeling [J]. CJTCMP, 2014, 29(6): 1994-1997. 